[19] B. S. Tsybakov and N. D. Vvedenskaya, "Stack algorithm for random multiple access," Probl. Peredach. Inform., vol. 16, no. 3,1980

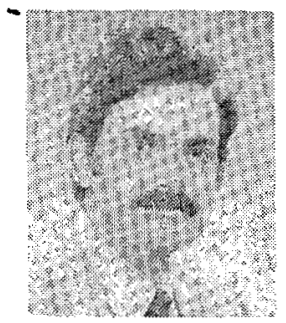

Izhak Rubin (S'69-M'71) was born in Haifa, Israel, on May 22, 1942. He received the B.Sc. and M.Sc. degrees in electrical engineering from the Technion-Israel Institute of Technology, Haifa, in 1964 and 1968, respectively, and the $\mathrm{Ph} . \mathrm{D}$. degree in electrical engineering from Princeton University, Princeton, NJ in 1970.

From 1964 to 1967 he served as an Engineer in the Israel Signal Corps where he worked on the analysis of communications systems. From 1967 to 1968 he was employed by the Israel Aircraft
Industry in the area of electronics and control engineering. During 1968 1970, he was.an RCA Fellow and Research Assistant in the Department of Electrical Engineering at Princeton University. Since 1970 he has been on the facultyof the Department of System Science, School of Engineering and Applied Science, University of California, Los Angeles, where he is currently a Professor. As a consultant to industry, he has been involved in the design and analysis of many communications and telecommunications systems and networks. He also serves as the President of IRI Corporation, a leading team of telecommunications and computer network experts who provide consulting and study services to industrial organizations. He served as the Co-Chairman of the 1981 IEEE International Symposium on Information Theory. His current interests are in the areas of telecommunication and computer networks, satellite communications, radio networks, communication systems, information theory, queueing systems, and stochastic processes.

Dr. Rubin is a member of Eta Kappa Nu.

\title{
Optimal Symbol-by-Symbol Detection for Duobinary Signaling
}

\author{
MITCHELL D. EGGERS AND JOHN H. PAINTER, SENIOR MEMBER, IEEE
}

\begin{abstract}
An optimal symbol-by-symbol detection scheme for duobinary signaling (Class I PRS) which exploits the inherent correlation properties of partial response signaling (PRS) is postulated. Analytical results indicate a maximum improvement of approximately $0.7 \mathrm{~dB}$ over conventional split shaping duobinary detection at a $10^{-4}$ error rate. Although duobinary signaling is emphasized, sufficient generality within the formulation is maintained to accommodate any class of PRS.
\end{abstract}

\section{INTRODUCTION}

A LTHOUGH a maximum likelihood detector has been shown to exhibit the lowest error rates for partial response detection [1], [2], the vast memory requirements and unwanted output delays prevent physical realization. An alternative detection scheme is postulated which exploits the prevailing correlation properties of partial response signaling (PRS), while avoiding the complexity encountered with maximum likelihood detection. The following discussion emphasizes duobinary (Class I PRS) signaling, yet preserves sufficient generality to accommodate any class of PRS.

\section{BACKGROUND}

\section{A. Precoded Duobinary Signaling}

Assuming the intersymbol interference is present only at adjacent sampling intervals, and in the absence of channel

Paper approyed by the Editor for Data Communication Systems of the IEEE Communications Society for publication without oral presentation. Manuscript received May 28, 1982; revised February 8, 1983.

The authors are with the Telecommunications and Control Systems Laboratory, Department of Electrical Engineering, Texas A\&M University, College Station, TX 77843. noise, the $k$ th precoded duobinary output symbol is given by [3]

$$
s_{k}=b_{k}+b_{k-1}
$$

where

$$
\begin{aligned}
& b_{k}=m_{k} \cdot b_{k-1} \\
& b_{k} \in\left\{\beta_{1}, \beta_{2}\right\}=\{-1,1\} \\
& s_{k} \in\left\{\delta_{1}, \delta_{2}, \delta_{3}\right\}=\{-2,0,2\} .
\end{aligned}
$$

Also, the source data stream $\left\{m_{k}\right\}$ in an analog $\{-1,1\}$ format is assumed to be equiprobable and independent. Defining the adjacent symbol correlation coefficient as

$$
\rho=\frac{E\left\{\left(s_{k}-\tilde{s}_{k}\right)\left(s_{k-1}-\bar{s}_{k-1}\right)\right\}}{\sigma_{s_{k}} \sigma_{s_{k-1}}}
$$

where

$$
\begin{aligned}
& \bar{s}_{j}=E\left\{s_{j}\right\} \\
& {\sigma_{s_{j}}}^{2}=\operatorname{var}\left\{s_{j}\right\},
\end{aligned}
$$

the statistical dependency of the output symbols formed from partial response filtering becomes apparent. The ensemble of joint output probabilities $\left\{p_{i j}\right\}$, where

$$
p_{i j}=P\left(s_{k}=\delta_{i}, s_{k-1}=\delta_{j}\right),
$$


TABLE I

JOINT OUTPUT PROBABILITIES

\begin{tabular}{|c|c|c|c|}
\hline$s_{k-1}^{s} k^{5}$ & -2 & 0 & 2 \\
\hline-2 & $1 / 8$ & $1 / 8$ & 0 \\
\hline 0 & $1 / 8$ & $1 / 4$ & $1 / 8$ \\
\hline 2 & 0 & $1 / 8$ & $1 / 8$ \\
\hline
\end{tabular}

necessary for the computation of $\rho$, are shown in Table I. The resulting correlation coefficient is shown to be $\rho=1 / 2$ [4]. Therefore, the adjacent received signal samples are 50 percent correlated although the source precoded symbols $\left\{b_{k}\right\}$ are statistically independent. Moreover, while precoding clearly removes the functional dependence of the output symbols on past symbol decisions, it preserves the statistical dependence with respect to the previous precoded bit.

\section{B. Channel Model}

The noise incurred in the channel is modeled as a zero mean, white Gaussian stochastic process with the power spectral density

$$
S_{w}(\omega)=\frac{N_{0}}{2} \mathrm{~W} / \mathrm{Hz}
$$

Hence, the received noise power within the Nyquist band $[-\pi / T, \pi / T]$ becomes

$$
P_{N}=2\left(\frac{N_{0}}{2}\right) \frac{1}{2 T}=\frac{N_{0}}{2 T} \mathrm{~W} .
$$

Due to the coloring of the PRS receiver filter, a statistical dependency is introduced within the noise process. This dependency is revealed from the autocorrelation function of the noise process at the detector $R_{n}(\tau)$ given by

$$
\begin{aligned}
R_{n}(\tau) & =F^{-1}\left\{S_{w}(\omega)\left|H_{R}(\omega)\right|^{2}\right\} \\
& =\frac{N_{0}}{2} F^{-1}\left\{\left|H_{R}(\omega)\right|^{2}\right\} .
\end{aligned}
$$

Considering the split shaping model, where the PRS pulse shaping filter $H(\omega)$ is apportioned equally between transmitter and receiver, the relationship for the receiver filter is $H_{R}(\omega)=$ $\sqrt{H(\omega)}$. Specifically, for duobinary signaling, $H(\omega)$ satisfies

$$
H(\omega)= \begin{cases}2 T \cdot \cos (\omega T / 2) & |\omega|<\pi / T \\ 0 & \text { elsewhere }\end{cases}
$$

where $T^{-1}$ is the symbol rate. Consequently, the autocorrelation function (6) of the noise process for duobinary signaling with split shaping is

$$
\begin{aligned}
R_{n}(\tau) & =\frac{N_{0}}{2} F^{-1}\{|H(\omega)|\}=\frac{N_{0}}{2} h(t) \\
& =\frac{N_{0}}{2} \cdot \frac{4}{\pi} \cos \frac{(\pi \tau / T)}{\left(1-4(\tau / T)^{2}\right)} .
\end{aligned}
$$

Hence, the noise autocorrelation function at the detector is the duobinary impulse scaled by the channel noise variance.

Evaluating the autocorrelation function at integer multiples of the sampling period $k T$ yields the covariance between any two noise samples separated by distance $k$. Examining (7), a significant amount of correlation is prevalent between adjacent noise samples $(\tau=k T=1 \cdot T)$, while beyond the neighboring sample $(k>1)$ the correlation appears negligible. Moreover, the adjacent noise samples are seen to be 33 percent correlated and the rate of correlation falls inversely with the square of the distance separating the samples. Consequently, only the correlation between adjacent noise samples is considered where the corrèlation coefficient $\left.\delta \triangleq \rho_{i j}\right|_{|i-j|=1}=$ $1 / 3$, thereby reducing the multivariate Gaussian noise density to a bivariate form given by

$$
p_{n_{i} n_{j}}(\alpha, \beta)=\frac{1}{2 \pi \sigma^{2} \sqrt{1-\xi^{2}}} \exp \left(\frac{-\left(\alpha^{2}-2 \xi \alpha \beta+\beta^{2}\right)}{2 \sigma^{2}\left(1-\xi^{2}\right)}\right)
$$

where

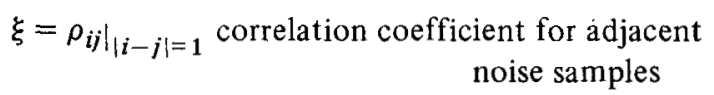

$\sigma^{2}=2 N_{0} / \pi \quad$ noise variance at the detector.

\section{OPTIMAL SYMBOL-BY-SYMBOL DETECTION}

To circumvent the complexity of maximum likelihood detection, a symbol-by-symbol detection operation is most desirable. The optimum symbol-by-symbol detector is defined as the detector which maximizes the a posteriori probability conditioned on the additional knowledge of the most recent symbol decision and noise sample. Assuming the most recent symbol decision is correct, the detector is then optimal with respect to the decision on $m_{k}$. The rationale for conditioning the decision event exclusively on the most recent symbol decision and noise sample is substantiated from the correlation properties discussed previously.

The primary assumption governing the derivation of the detector is perfect synchronization of the PRS waveform. As a result, the $k$ th sample input to the detector will be denoted

$$
r_{k}=s_{k}+n_{k} \text {. }
$$

With the prescribed modeling of the detector input, the conditioning events become

$$
\begin{array}{ll}
r_{k}=\gamma_{k} & \text { present received sample } \\
r_{k-1}=\gamma_{k-1} & \text { most recent received sample } \\
\hat{s}_{k-1}=\delta_{i} & \text { most recent symbol decision. }
\end{array}
$$


The derivation of the optimal symbol-by-symbol detector follows from maximizing the a posteriori probability conditioned on the above events. Hence, the detector sets $\hat{s}_{k}=\delta_{l}$ if and only if

$$
\begin{gathered}
P\left(s_{k}=\delta_{l} \mid r_{k}=\gamma_{k}, r_{k-1}=\gamma_{k-1}, \hat{s}_{k-1}=\delta_{i}\right) \\
>P\left(s_{k}=\delta_{j} \mid r_{k}=\gamma_{k}, r_{k-1}=\gamma_{k-1}, \hat{s}_{k-1}=\delta_{i}\right) \\
\text { all } j \neq l .
\end{gathered}
$$

Rewriting the decision rule in terms of the known a priori probabilities (Table I) and density functions, the maximum a posteriori detector sets $\hat{s}_{k}=\delta_{l}$ whenever

$$
\begin{gathered}
p_{n_{k-1}, n_{k}}\left(\gamma_{k-1}-\delta_{i}, \gamma_{k}-\delta_{l}\right) P\left(\delta_{i}, \delta_{l}\right) \\
>p_{n_{k-1}, n_{k}}\left(\gamma_{k-1}-\delta_{i}, \gamma_{k}-\delta_{j}\right) P\left(\delta_{i}, \delta_{j}\right) \\
\text { all } j \neq l .
\end{gathered}
$$

With the specification of the bivariate Gaussian noise process (8) the detector chooses $\hat{s}_{k}=\delta_{l}$ if and only if

$$
\begin{gathered}
P\left(\delta_{i}, \delta_{l}\right) \exp -\left[\frac{\delta_{l}^{2}+\delta_{l}\left(2 \xi\left(\gamma_{k-1}-\delta_{i}\right)-2 \gamma_{k}\right)}{2 \sigma^{2}\left(1-\xi^{2}\right)}\right] \\
>P\left(\delta_{i}, \delta_{j}\right) \exp -\left[\frac{\delta_{j}^{2}+\delta_{j}\left(2 \xi\left(\gamma_{k-1}-\delta_{i}\right)-2 \gamma_{k}\right)}{2 \sigma^{2}\left(1-\xi^{2}\right)}\right] \\
\text { all } j \neq l .
\end{gathered}
$$

A realization of the decision rule (13) yields a variable threshold slicer, where the threshold levels are given by [4] .

$$
\begin{aligned}
f_{i}\left(\gamma_{k-1}\right)= & \frac{\delta_{j}+\delta_{l}}{2}+\xi\left(\gamma_{k-1}-\delta_{i}\right) \\
& +\frac{\sigma^{2}\left(1-\xi^{2}\right)}{\left(\delta_{j}-\delta_{l}\right)} \ln \frac{P\left(\delta_{i}, \delta_{l}\right)}{P\left(\delta_{i}, \delta_{j}\right)}
\end{aligned}
$$

That is, to yield a decision $\hat{s}_{k}$, the value of the present received sample value $\gamma_{k}$ is compared to a threshold that is function. ally dependent upon the past received sample value $\gamma_{k-1}$. Notice that the inclusion of the noise correlation properties within the conditioning event is seen to translate the threshold level (14) by an amount equal to the previous noise sample estimate $\left(\hat{n}_{k-1}=r_{k-1}-\hat{s}_{k-1}\right)$, weighted by the adjacent noise correlation coefficient. Second, the conditioning provided by the PRS signal correlation adjusts the levels according to the joint probability of the output symbols within the correlation span $\left(s_{k-1}, s_{k}\right)$.

Depending upon the most recent symbol decision, $\hat{s}_{k-1}$, the threshold levels (14) can be evaluated in terms of the most recent received sample $\left(\gamma_{k-1}\right)$ and the noise variance. Thus, three cases arise from the permissible duobinary output levels in which the specific thresholds are

$$
\begin{aligned}
& \text { Case I: } \hat{s}_{k-1}=\delta_{1}=-2 \\
& f_{1}\left(\gamma_{k-1}\right)=-1+1 / 3\left(\gamma_{k-1}+2\right) .
\end{aligned}
$$

Case II: $\hat{s}_{k-1}=\delta_{2}=0$

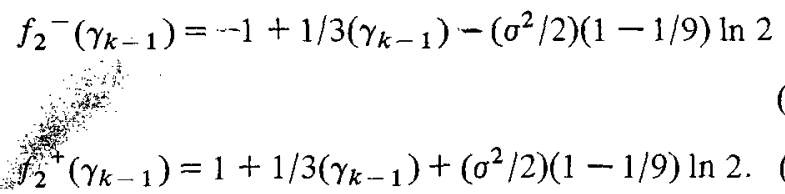

Case III: $\hat{s}_{k-1}=\delta_{3}=2$

$f_{3}\left(\gamma_{k-1}\right)=1+1 / 3\left(\gamma_{k-1}-2\right)$.

The probabilistic behavior of the detector input sequence $\left\{r_{k}\right\}$ is depicted according to the various cases in Fig. 1. The a posteriori probabilities are shown as a function of the present received sample value $\gamma_{k}$. The decision regions denoted $I_{i j}$, where

$$
\begin{aligned}
& i \in\{1,2,3\} ; \quad \text { case selector } \\
& j=\left\{\begin{array}{ll}
0 & \hat{m}_{k}=-1 \\
1 & \hat{m}_{k}=1
\end{array} ;\right. \text { message selector }
\end{aligned}
$$

partition the ternary received sampled space into the disjoint binary message regions $\left(\hat{m}_{k}=-1, \hat{m}_{k}=1\right)$, in accordance with (1). Consequently, the decision-directed detector with noise feedback is a variable threshold slicer, controlled by both the most recent symbol decision $\left(\hat{s}_{k-1}\right)$ and the previous noise sample estimate $\left(\hat{n}_{k-1}\right)$. Two other detectors, being derivatives of the preceding, will be developed to reveal the degree of detection improvement achieved by considering the correlation properties of the received signal and noise samples.

Considering the correlation of the signal samples only, the decision-directed detector is identical with the preceding detector with the exception of the noise feedback. Thus, the a posteriori probabilities are conditioned only on the knowledge of the most recent symbol decision $\left(s_{k-1}\right)$, resulting in a detector which sets $\hat{s}_{k}=\delta_{l}$ if and only if

$$
\begin{aligned}
& P\left(s_{k}=\delta_{l} / r_{k}=\gamma_{k}, s_{k-1}=\delta_{i}\right) \\
& \quad>P\left(s_{k}=\delta_{j} / r_{k}=\gamma_{k}, s_{k-1}=\delta_{i}\right) \quad \text { all } j \neq l .
\end{aligned}
$$

Now, the Gaussian noise density is single-dimensional since the noise process is considered statistically independent. With this density substitution, the decision-directed detector chooses $\hat{s}_{k}=\delta_{l}$ whenever

$$
\begin{aligned}
& P\left(\delta_{i}, \delta_{l}\right) \exp -\left[\frac{\delta_{l}{ }^{2}-2 \delta_{l} \gamma_{k}}{2 \sigma^{2}}\right] \\
& >P\left(\delta_{i}, \delta_{j}\right) \exp -\left[\frac{\delta_{j}{ }^{2}-2 \delta_{j} \gamma_{k}}{2 \sigma^{2}}\right] \quad \text { all } j \neq l
\end{aligned}
$$

Realizing (17) in the form of a variable threshold slicer, the thresholds are given by

$$
f_{i}^{\prime}=\frac{\delta_{j}+\delta_{l}}{2}+\frac{\sigma^{2}}{\delta_{j}-\delta_{l}} \ln \frac{P\left(\delta_{i}, \delta_{l}\right)}{P\left(\delta_{i}, \delta_{j}\right)}
$$




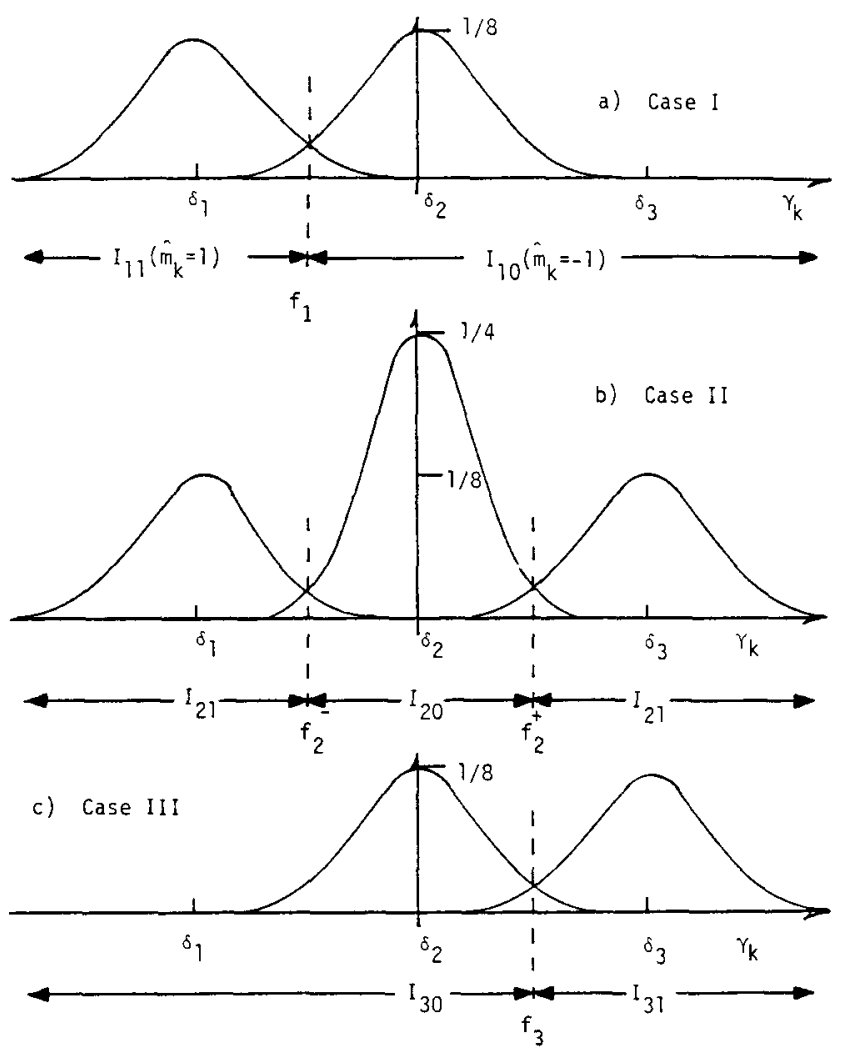

Fig. 1. Probabilistic behavior of the received samples according to (a) Case I, (b) Case II, (c) Case III.

which are equivalent to the levels given by (14) with the noise correlation coefficient $\xi$ set to zero. In an analogous manner, three cases arise where the specific thresholds are given by

Case I: $\quad f_{1}{ }^{\prime}=-1$.

Case II: $\quad f_{2}^{\prime-}=-1-\frac{\sigma^{2}}{2} \ln 2$

$$
f_{2}{ }^{++}=1+\frac{\sigma^{2}}{2} \ln 2
$$

Case III: $\quad f_{3}{ }^{\prime}=+1$.

Therefore, the decision-directed detector is a variable threshold slicer controlled by the most recent symbol decision.

The final derivative of the decision-directed detector with noise feedback is the mean detector. This detector ignores the correlation properties of both the signal and noise samples and simply chooses $\hat{s}_{k}=\delta_{l}$ whenever

$$
P\left(s_{k}=\delta_{l} / r_{k}=\gamma_{k}\right)>P\left(s_{k}=\delta_{j} / r_{k}=\gamma_{k}\right) \quad \text { all } j \neq l .
$$

Thus, the resulting mean detector threshold levels do not discriminate with regard to the permissible cases; rather, the levels are based on the average output symbol occurrence. The two threshold levels for the mean detector are given by

$$
f-=-1-\frac{\sigma^{2}}{2} \ln 2
$$

$$
f+=1+\frac{\sigma^{2}}{2} \ln 2
$$

and are seen to be functionally dependent only upon the noise variance.

For comparison purposes, the conventional detector for split shaping duobinary signaling will also be considered. This detector is simply a fixed level slicer with the symmetric thresholds placed at -1 and +1 to partition the ternary signal space.

For convenience, the detectors will be henceforth denoted by the following acronyms:

$\begin{array}{ll}\mathrm{CD} / \mathrm{S} & \text { conventional detector for split shaping } \\ \mathrm{MD} & \text { mean detector } \\ \mathrm{D}^{3} & \text { decision-directed detector } \\ \mathrm{D}^{3} / \mathrm{N} & \text { decision-directed detector with noise feedback }\end{array}$

Finally, the distinction among other PRS optimum detector derivations is credited to the a posteriori conditioning event. This event is determined from the degree of correlation present in the noise samples, due to receiver filtering, and within the signal samples themselves.

\section{PROBABILITY OF ERROR AND COMPARISON WITH OTHER DETECTION METHODS}

\section{A. Probability of Error and Comparison for a Known Channel Level}

Concerning the decision-directed detectors, the most recent decision $\hat{s}_{k-1}$ is assumed correct. This assures tractable error probability expressions by establishing proper thresholds for the current decision $\hat{s}_{k}$. Hence, the resulting $P(e)$ expressions for the $D^{3} / N$ and $D^{3}$ actually represent a lower bound on probability of error.

The increased conditioning of the $D^{3} / N$, based on the correlation present in the information bearing sequence $\left\{s_{k}\right\}$ and the noise samples $\left\{n_{k}\right\}$, is incorporated into the $P(e)$ expression based on the average occurrence of such events. Therefore, the average error probability over the ensemble of possible $\left\{s_{k}\right\}$ and $\left\{n_{k}\right\}$ occurrences within the correlation span yields

$$
P(e)=\sum_{i} \sum_{j} P\left(e / s_{k-1}=\delta_{i}, s_{k}=\delta_{j}\right) P\left(s_{k-1}=\delta_{i}, s_{k}=\delta_{j}\right)
$$

where

$$
\begin{aligned}
& P\left(e / s_{k-1}=\delta_{i}, s_{k}=\delta_{j}\right) \\
& \quad=E\left\{P\left(e / s_{k-1}=\delta_{i}, s_{k}=\delta_{j}, r_{k-1}\right\}\right. \\
& \quad=\int_{-\infty}^{\infty} P\left(e / s_{k-1}=\delta_{i}, s_{k}=\delta_{j}, r_{k-1}=\alpha\right) p_{r_{k-1}}(\alpha) d \alpha
\end{aligned}
$$

and

$$
(e) \Leftrightarrow\left(\hat{n}_{k} \neq m_{k}\right)
$$

The conditional probability (23) arises from the conditioning 
zvent on the previously determined noise sample $\left(\hat{n}_{k-1}=\right.$ $\left.r_{k-1}-\hat{s}_{k-1}\right)$. Thus, to reflect the added conditioning, the conditional probability (23) is averaged with respect to the permissible range of the random variable $r_{k-1}$. The conditional probability within the integral (23),

$$
P\left(e / \delta_{i}, \delta_{j}, \alpha\right)=\sum_{m} \int_{f_{m}(\alpha)}^{\infty} N\left(\delta_{j}+\xi\left(\alpha-\delta_{i}\right), \sigma \sqrt{1-\xi^{2}}\right)
$$

where $N(x, y)$ represents a Gaussian density with mean $x$ and variance $y^{2}$, is functionally independent of the most recent received sample value $\alpha$. Therefore, (23) reduces to

$$
\begin{aligned}
& P\left(e / s_{k-1}=\delta_{i}, s_{k}=\delta_{j}\right) \\
& \quad=P\left(e / s_{k-1}=\delta_{i}, s_{k}=\delta_{j}, r_{k-1}=\alpha\right) \int_{-\infty}^{\infty} p_{y_{j}-1}(\alpha) d \alpha \\
& \quad=\sum_{m} Q\left[\frac{f_{m}(\alpha)-\left\{\delta_{j}+\xi\left(\alpha-\delta_{i}\right)\right\}}{\sigma \sqrt{1-\xi^{2}}}\right]
\end{aligned}
$$

where

$$
Q(x)=\frac{1}{\sqrt{2 \pi}} \int_{x}^{\infty} e^{-t^{2} / 2} d t .
$$

The above sum indexed on $m$ includes all error events, represented as intervals bounded by the thresholds $f_{m}(\alpha)$ (see Fig. $1)$. The independence is explained by examining the conditional mean of the density function in (24)

$$
\mu_{n_{k} / n_{k-1}}=\delta_{j}+\xi\left(\alpha-\delta_{i}\right)
$$

and the threshold functions $f_{m}(\alpha)(14)$. Any translation of the density function by an amount $\xi\left(\alpha-\delta_{i}\right)$ is countered by an equivalent translation in the threshold value. Hence, the conditional error probability (24) is independent of the previous received sample $r_{k-1}=\alpha$, due to the counteraction of the thresholds.

Inserting the joint probabilities of the adjacent output symbols tabulated in Table $\mathrm{I}$, the $P(e)$ expression (22) becomes

$$
P(e)=\sum_{i=1}^{3} P_{i}(e)
$$

where

$$
\begin{aligned}
P_{1}(e)= & \frac{1}{8} P\left(e / \delta_{1}, \delta_{1}\right)+\frac{1}{8} P\left(e / \delta_{1}, \delta_{2}\right) \\
P_{2}(e)= & \frac{1}{8} P\left(e / \delta_{2}, \delta_{1}\right)+\frac{1}{8} P\left(e / \delta_{2}, \delta_{3}\right) \\
& +\frac{1}{4} P\left(e / \delta_{2}, \delta_{2}\right) \\
P_{3}(e)= & \frac{1}{8} P\left(e / \delta_{3}, \delta_{2}\right)+\frac{1}{8} P\left(e / \delta_{3}, \delta_{3}\right) .
\end{aligned}
$$

Clearly, each $P_{i}(e)$ represents the average probability of error for the $i$ th case encountered. The resulting probabilities
$\left\{P_{i}(e)\right\}$ according to the respective cases are given by (see Fig. 1)

Case I: $s_{k-1}=\delta_{1}=-2$

$$
\begin{aligned}
P_{1}(e)= & \frac{1}{8} \int_{f_{1}(\alpha)}^{\infty} N\left(-2+\xi(\alpha+2), \sigma_{0}\right) \\
& +\frac{1}{8} \int_{-\infty}^{f_{1}(\alpha)} N\left(0+\xi(\alpha+2), \sigma_{0}\right)
\end{aligned}
$$

where

$$
\sigma_{0}=\sigma \sqrt{1-\xi^{2}} .
$$

Case II: $s_{k-1}=\delta_{2}=0$

$$
\begin{aligned}
P_{2}(e)= & \frac{1}{8} \int_{f_{2}-(\alpha)}^{f_{2}+(\alpha)} N\left(-2+\xi(\alpha-0), \sigma_{0}\right) \\
& +\frac{1}{8} \int_{f_{2}-(\alpha)}^{f_{2}+(\alpha)} N\left(2+\xi(\alpha-0), \sigma_{0}\right) \\
& +\frac{1}{4} \int_{-\infty}^{f_{2}-(\alpha)} N\left(0+\xi(\alpha-0), \sigma_{0}\right) \\
& +\int_{f_{2}+(\alpha)}^{\infty} N\left(0+\xi(\alpha-0), \sigma_{0}\right) .
\end{aligned}
$$

Case III: $s_{k-1}=\delta_{3}=2$

$$
\begin{aligned}
P_{3}(e)= & \frac{1}{8} \int_{f_{3}(\alpha)}^{\infty} N\left(0+\xi(\alpha-2), \sigma_{0}\right) \\
& +\frac{1}{8} \int_{-\infty}^{f_{3}(\alpha)} N\left(2+\xi(\alpha-2), \sigma_{0}\right)
\end{aligned}
$$

Combining $\left\{P_{i}(e)\right\}$ for the three cases in conjunction with (27), the average probability of single bit error is [4]

$$
\begin{aligned}
P(e)= & \frac{1}{2}\left[Q\left(\frac{1}{\sigma_{0}}\right)+Q\left(\frac{1+\Delta_{0}}{\sigma_{0}}\right)\right] \\
& +\frac{1}{4}\left[Q\left(\frac{1-\Delta_{0}}{\sigma_{0}}\right)-Q\left(\frac{3+\Delta_{0}}{\sigma_{0}}\right)\right]
\end{aligned}
$$

where

$$
\begin{aligned}
& \Delta_{0}=\Delta\left(1-\xi^{2}\right) \\
& \Delta=\sigma^{2} \ln 2 / 2 .
\end{aligned}
$$

Since the $D^{3}$ is analogous to the $D^{3} / N$ with the exception of the noise feedback, whereby only the correlation of the information sequence $\left\{s_{k}\right\}$ is considered, the $P(e)$ expression follows from (32) with $\xi=0$. Thus, the average probability of 
single bit error for the $\mathrm{D}^{3}$ is given by

$$
\begin{aligned}
P(e)= & \frac{1}{2}\left\{Q\left(\frac{1}{\sigma}\right)+Q\left(\frac{1+\Delta}{\sigma}\right)\right\} \\
& +\frac{1}{4}\left\{Q\left(\frac{1-\Delta}{\sigma}\right)-Q\left(\frac{3+\Delta}{\sigma}\right)\right\} .
\end{aligned}
$$

Due to the neglect of the correlation present within the information and noise sequences $\left\{s_{k}\right\}$ and $\left\{n_{k}\right\}$, respectively, the error event for the MD is only conditioned on the current transmitted symbol. Thus, the $P(e)$ is formulated according to the total probability

$$
P(e)=\sum_{i} P\left(e / s_{k}=\delta_{i}\right) P\left(s_{k}=\delta_{i}\right)
$$

Here, the thresholds vary only with respect to the noise variance $\sigma^{2}$ at the detector. The resulting probability of single bit error for the MD is

$$
P(e)=Q\left(\frac{1+\Delta}{\sigma}\right)+\frac{1}{2}\left\{Q\left(\frac{1-\Delta}{\sigma}\right)-Q\left(\frac{3+\Delta}{\sigma}\right)\right\}
$$

The final detector, the $\mathrm{CD} / \mathrm{S}$, is a fixed level slicer with the thresholds placed symmetrically at -1 and +1 . Therefore, the $\mathrm{CD} / \mathrm{S}$ is a special case of the MD with the stipulation $\Delta=$ 0 . Consequently, substitution of $\Delta=0$ into (37) yields the probability of single bit error for the $\mathrm{CD} / \mathrm{S}$.

$$
P(e)=\frac{3}{2} Q(1 / \sigma)-\frac{1}{2} Q(3 / \sigma) .
$$

The asymptotic approximations for the $P(e)$ expressions, obtained by allowing the SNR to become sufficiently large $(\Delta \rightarrow 0)$, are given by

$$
\begin{array}{ll}
\mathrm{D}^{3} \mathrm{~N}: & P(e) \cong \frac{5}{4} Q\left(1 / \sigma \sqrt{1-\xi^{2}}\right) \\
\mathrm{D}^{3}: & P(e) \cong \frac{5}{4} Q(1 / \sigma) \\
\mathrm{MD}: & P(e) \cong \frac{3}{2} Q(1 / \sigma) \\
\mathrm{CD} / \mathrm{S}: & P(e) \cong \frac{3}{2} Q(1 / \sigma) .
\end{array}
$$

Notice that the asymptotic performance of the detection process is improved by decision-direction. The additive detection improvement provided by the noise feedback is revealed by the presence of the correlation term $\sqrt{1-\xi^{2}}$ in the $Q$ function argument. Also from the asymptotic approximations, the $\mathrm{MD}$ and $\mathrm{CD} / \mathrm{S}$ are shown to perform identically.

For split shaping duobinary signaling, the averaging channel $\operatorname{SNR}\left(P_{s} / P_{n}\right)$ is given by [5]

$$
\mathrm{SNR}=\frac{8}{\pi N_{0}}
$$

Here, $P_{s}$ and $P_{n}$ respectively denote the average symbol power and noise power in the channel. Expressing the noise variance

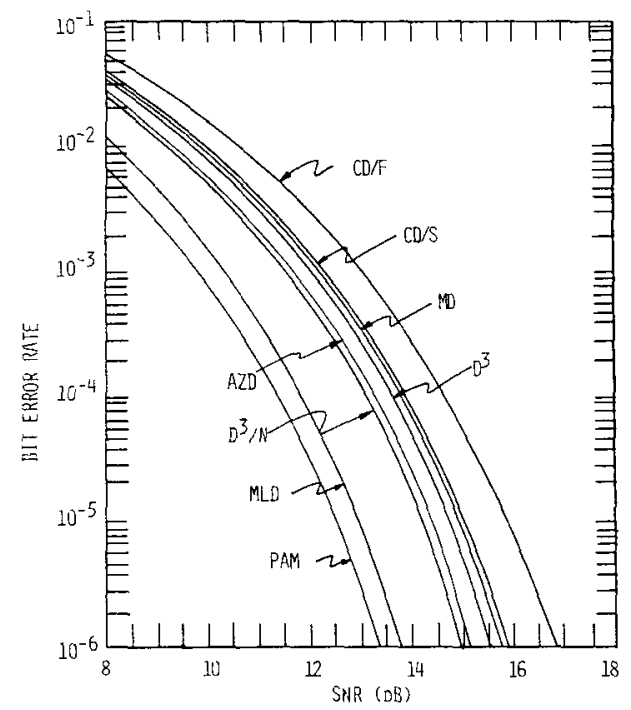

Fig. 2. Low error rate performance of the various detection schemes.

$\sigma^{2}=2 N_{0} / \pi$ at the detector in terms of the SNR results in the bit error rate curves shown in Fig. 2 . The additional acronyms include:

$\mathrm{CD} / \mathrm{F}$ conventional detector for full transmitter shaping

PAM ideal pulse amplitude modulation with split shaping

MLD maximum likelihood detector

accompanied by the following asymptotic $P(e)$ expressions [6], [7].

$$
\mathrm{CD} / \mathrm{F}: \quad P(e) \cong \frac{3}{2} Q\left(\sqrt{\frac{\mathrm{SNR}}{2}}\right)
$$

PAM: $\quad P(e)=Q(\sqrt{\mathrm{SNR}})$

The $P(e)$ curve shown in Fig. 2 for maximum likelihood detection results from simulation studies [2].

Notice that within the asymptotic range ( $S N R>8 \mathrm{~dB}$ ), both the MD and $\mathrm{D}^{3}$ differ only marginally from the $\mathrm{CD} / \mathrm{S}$. However, with the inclusion of the noise feedback, the resulting $\mathrm{D}^{3} / \mathrm{N}$ is shown to exhibit an improvement of approximately $0.7 \mathrm{~dB}$ with respect to the $\mathrm{CD} / \mathrm{S}$ at a $10^{-4}$ error rate. For the lower range (SNR $<7 \mathrm{~dB}$ ), the $\mathrm{D}^{3} / \mathrm{N}$ margin of improvement increases to approximately $1 \mathrm{~dB}$.

In comparison to maximum likelihood detection, which exhibits the lowest attainable $P(e)$ for duobinary signaling, the $\mathrm{D}^{3} / \mathrm{N}$ suffers $1.3 \mathrm{~dB}$ at a $10^{-4}$ error rate. Hence, the $\mathrm{D}^{3} / \mathrm{N}$ avoids the complexity associated with maximum likelihood detection at an expense of at least $1.3 \mathrm{~dB}$ SNR degradation.

An alternate approach to improving PRS detection that avoids the constraints imposed by maximum likelihood detection is null zone or ambiguity zone detection, postulated by Smith [8]. Essentially, detection is performed by a quantizer with ambiguity levels about the threshold regions. Most received samples which fall into the ambiguity or null zones are replaced by the correct decision value based on the partial response signal redundancy. Nulls which cannot be replaced 
unambiguously are substituted by the most probable values based on the previous received sample values: The analysis by Smith indicates an improvement of at least $0.7 \mathrm{~dB}$ at a $10^{-4}$ error rate over conventional duobinary detection with split shaping employed. The inability to determine the exact improvement of this scheme with the split shaping model is attributed to the assumption of independent noise samples at the detector, which is valid only for full shaping. That is, the improvement of $0.7 \mathrm{~dB}$ was obtained analytically for full transmitter shaping and interpreted as an upper performance bound for split shaping.

The question of the optimal null zone partitioning for such a scheme was pursued by Kobayashi and Tang [9]. Extending the works of Smith from an algebraic standpoint, an optimal ambiguity zone was established (optimal in the sense of minimizing the probability of ambiguous reception given the probability of an incorrect decision). The formulation revealed the functional dependence of the optimal ambiguity zone upon the noise variance. The performance analysis (assuming independent noise samples at the detector and correct previous decisions) led to the following asymptotic $P(e)$ expression for optimal ambiguity zone detection (AZD) [9] .

$$
\mathrm{AZD}: P(e) \cong \frac{3}{2} Q(2(\sqrt{2}-1) \sqrt{\mathrm{SNR}})
$$

Several factors must be recognized to warrant correct interpretation of the relative performance of the $\mathrm{D}^{3} / \mathrm{N}$ and the AZD method illustrated in Fig. 2. First, both $P(e)$ expressions represent upper performance bounds due to the assumption of correct previous decisions. Second, the $D^{3} / N$ analysis accommodates correlated noise samples at the detector and hence accurately reflects split shaping. However, the AZD analysis assumes independent noise samples at the detector and therefore accurately depicts full shaping. Conceivably, from the bounds obtained by Smith [8], AZD incorporated with split shaping would increase the margin of improvement beyond $0.7 \mathrm{~dB}$.

\section{B. Probability of Error for an Unknown Channel Level}

Suppose the detector input signal is of the form

$$
r_{k}=a s_{k}+n_{k}
$$

where " $a$ " represents a fixed but unknown channel level. The channel level constant is assumed to be statistically independent of both the transmitted signal and channel noise.

The corresponding decision rule, which maintains the optimality as previously defined, is realized by a variable threshold detector with a threshold function given by

$$
\begin{aligned}
f_{i}\left(\gamma_{k-1} ; a\right)= & a \frac{\left(\delta_{j}+\delta_{l}\right)}{2}+\xi\left(\gamma_{k-1}-a \delta_{i}\right) \\
& +\frac{a^{2}\left(1-\xi^{2}\right)}{a\left(\delta_{j}-\delta_{l}\right)} \ln \frac{P\left(\delta_{i}, \delta_{l}\right)}{P\left(\delta_{i}, \delta_{j}\right)}
\end{aligned}
$$

The derivation of (48) follows analogously from the previous case whereby the channel level was assumed to be unity. Con- sequently, due to the dependency of the threshold function upon the channel level, automatic gain control (AGC) circuitry must accommodate the threshold detector to maintain optimality.

The probability of error expression resulting from the unknown channel level signal model (47) and the optimal threshold function (48) is given by

$$
\begin{aligned}
P(e)= & \frac{1}{2}\left\{Q\left(\frac{a}{\sigma_{0}}\right)+Q\left(\frac{a+\Delta_{0} / a}{\sigma_{0}}\right)\right\} \\
& +\frac{1}{4}\left\{Q\left(\frac{a-\Delta_{0} / a}{\sigma_{0}}\right)-Q\left(\frac{3 a+\Delta_{0} / a}{\sigma_{0}}\right)\right\} .
\end{aligned}
$$

Notice that the utilization of the threshold function (48) in determining the $P(e)$ imposes the additional constraint of perfect AGC. The asymptotic approximation for the above $P(e)$ expression is

$$
P(e) \cong \frac{5}{4} Q\left(a / \dot{\sigma}_{0}\right)
$$

while the asymptotic $P(e)$ expression obtained in the absence of $\operatorname{AGC}\left(f\left(\gamma_{k-1} ; a\right)=f\left(\gamma_{k-1} ; 1\right)\right)$ is given by

$$
\begin{aligned}
P_{0}(e) \cong & \frac{1}{2} Q\left(\frac{1}{\sigma_{0}}\right)+\frac{1}{4}\left[Q\left\{\frac{2(a-1)(1-\xi)+1}{\sigma_{0}}\right\}\right. \\
& \left.+Q\left\{\frac{2 \xi(a-1)+1}{\sigma_{0}}\right\}\right]+\frac{1}{4}\left[Q\left\{\frac{2 a-1}{\sigma_{0}}\right\}\right. \\
& \left.-Q\left\{\frac{2 a+1}{\sigma_{0}}\right\}\right] .
\end{aligned}
$$

Clearly, AGC employed in the decision process enhances the $P(e)$ performance.

Most importantly, however, is the question of sensitivity. Since perfect $\dot{A} G C$ is not physically obtainable, it is desirable to determine the amount of bit error rate performance sacrificed due to the inability of the AGC to recover the exact channel level constant. Thus, the variation of the bit error rate with respect to small deviations about the exact channel level constant is examined.

Consider a bit error rate measure of sensitivity defined according to

$$
\eta=\frac{P_{\epsilon}(e)-P(e)}{P_{\epsilon}(e)}
$$

where

$$
\begin{aligned}
& P(e)=\text { probability of error assuming perfect AGC } \\
& P_{\epsilon}(e)=\text { probability of error with AGC error } \epsilon .
\end{aligned}
$$

That is, the sensitivity is a normalized measure of error rate difference between the ideal case which presumes perfect AGC and the physical case where an error $\epsilon$ exists between the AGC 


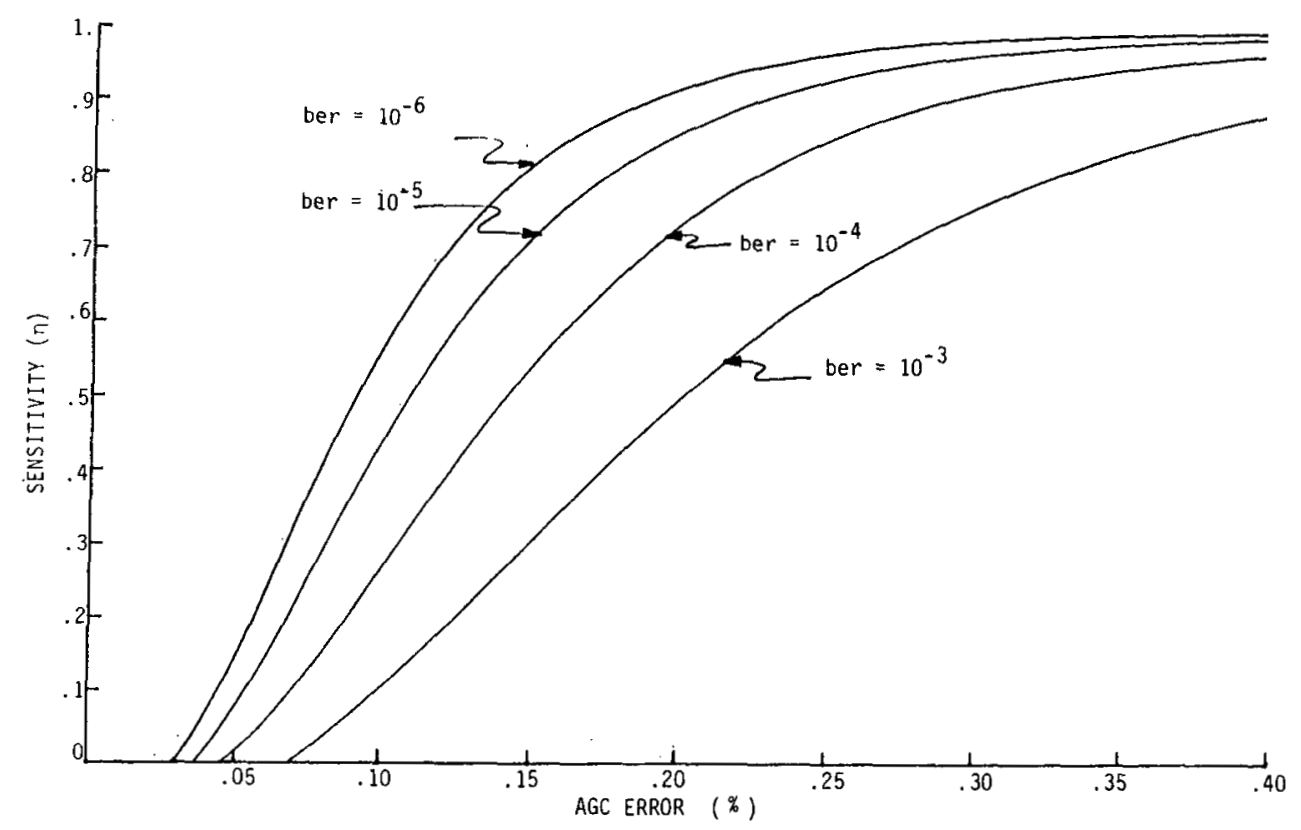

Fig. 3. Performance sensitivity with respect to AGC error for various error rates.

determined channel level constant and the actual value. The derivation of the $P_{\epsilon}(e)$ follows the previous work with the exception that the threshold function $f_{i}^{\epsilon}\left(\gamma_{k-1} ; \mu\right)$ now encompasses the discrepancy between the actual and AGC determined channel level constant according to

$$
f_{i}^{\epsilon}\left(\gamma_{k-1} ; \mu\right)=f_{i}\left(\gamma_{k-1} ; \dot{a}+\epsilon\right)
$$

Consequently, the asymptotic $P_{\epsilon}(e)$ becomes

$$
\begin{aligned}
P_{\epsilon}(e) \cong & \frac{1}{4}\left[Q\left\{\frac{a-\epsilon(1-2 \xi)}{\sigma_{0}}\right\}+Q\left\{\frac{\dot{a}+\epsilon(1-2 \xi)}{\sigma_{0}}\right\}\right] \\
& +\frac{1}{4} Q\left(\frac{a-\epsilon}{\sigma_{0}}\right)+\frac{1}{2} Q\left(\frac{a+\epsilon}{\sigma_{0}}\right) .
\end{aligned}
$$

Hence, for sufficiently large SNR's, the sensitivity expression (52) utilizing (50) and (54) is

$$
\eta \cong \mathrm{i}-5 a\left(a^{2}-\epsilon^{2} / a\right) \cdot\left(a^{2}-\epsilon^{2}\right) \cdot h^{-1}(a, \epsilon)
$$

where

$$
\begin{aligned}
h(a, \epsilon) & \\
= & (a+\epsilon / 3)\left(a^{2}-\epsilon^{2}\right) \exp \left\{-\frac{1}{2} \sigma_{0}^{2}\left(\frac{-2 a \epsilon}{3}+\epsilon^{2} / 9\right)\right\} \\
& +(a-\epsilon / 3)\left(a^{2}-\epsilon^{2}\right) \exp \left\{-\frac{1}{2} \sigma_{0}^{2}\left(\frac{2 \dot{a} \epsilon}{3}+\epsilon^{2} / 9\right)\right\} \\
& +\left(a^{2}-\epsilon^{2} / 9\right)(a+\epsilon) \exp \left\{-\frac{1}{2} \sigma_{0}{ }^{2}\left(-2 a \epsilon+\epsilon^{2}\right)\right\} \\
& +\left(a^{2}-\epsilon^{2} / 9\right)(a-\epsilon) \exp \left\{-\frac{1}{2} \sigma_{0}{ }^{2}\left(2 a \epsilon+\epsilon^{2}\right)\right\}
\end{aligned}
$$

and the exponential approximation $Q(\alpha) \cong(1 / 2 \pi \alpha) \exp \left(-\alpha^{2} / 2\right)$ has been employed. Fig. 3 depicts the family of sensitivity curves generated by (55) for a substantial range of error rates. The actual channel level was assumed to be unity and as a result, the AGC error is expressed as a percentage difference. For example, suppose a given AGC unit exhibits an average error of 11 percent. Then from Fig. 3, together with an ideal bit error rate of $10^{-5}$, the sacrifice in performance would be a doubling of the error rate.

\section{CONCLUSION}

By exploiting the prevailing correlation of the induced intersymbol interference inherent to partial response schemes, an optimal symbol-by-symbol detection scheme resulted. This scheme was shown to be dependent upon the channel level. Furthermore, the analytical $P(e)$ expression revealed a maximum improvement of approximately $0.7 \mathrm{~dB}$ in SNR over conventional duobinary detection with split shaping. However, with respect to maximum likelihood detection, the optimal symbol-by-symbol detection scheme suffered $1.3 \mathrm{~dB}$ at an error rate of $10^{-4}$.

\section{REFERENCES}

[1] H. Kobayashi, "Correlative level coding and maximum-likelihood decoding,' IEEE Trans. Inform. Theory, vol. IT-17, pp. 586-594, Sept. 1971.

[2] G. D. Forney, Jr., "Maximum-likelihood sequence estimation of digital sequences in the presence of intersymbol interference," IEEE Trans. Inform. Theory, vol. IT-18, pp. 363-378, May 1972.

[3] A. Lender, "The duobinary technique for high speed data transmission," IEEE Trans. Commun. Electron., vol. 82, pp. 214-218, May 1963.

[4] M. D. Eggers, "The development of a decision directed modem for quadrature duobinary signaling," M.S. thesis, Texas A\&M Univ., College Station, Aug. 1981.

[5] P. Kabal and S. Pasupathy, "Partial-response signaling," IEEE Trans. Commun., vol. COM-23. pp. 921-934. Sept. 1975.

[6] K. S. Shanmugan, Digital and Analog Communication Systems. New York, Wiley, 1979.

[7] R. Lucky, J. Salz, and E. Weldon, Jr., Principles of Data Communications. New York: McGraw-Hill, 1968.

[8] J. W. Smith, "Error control in duobinary data systems by means of null zone detection," IEEE Trans. Commun. Technol., vol. COM16, pp. 825-830, Dec. 1968. 
[9] H. Kobayashi and D. Tang, "On decoding of correlation level coding systems with ambiguity zone detection," IEEE Trans. Commun. Technol., vol. COM-19, pp. 467-477, Aug. 1971.

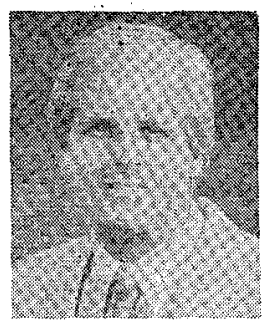

Mitchell D. Eggers was born in Jefferson City, MO, in 1958. He received the B.S. (with honors) and M.S. degrees in electrical engineering from Texas A \& M University, College Station, in 1980 and 1981 , respectively.

In 1979, he served as an Assistant at Texas A \& M University where he conducted research in ionospheric range error estimation. Also, from 1979 to 1981 , he was an Engineering Consultant for Arthur A. Collins, Inc., Dallas, TX, where he was involved in the research of PRS communication systems. Presently, he is employed as a Lecturer at Texas A \& M University while pursuing the Ph.D. degree in electrical engineering, specializing in optimal adaptive estimation.

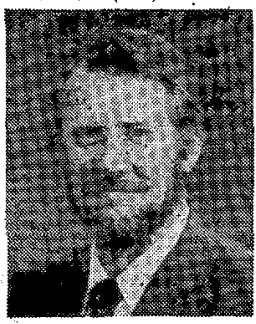

John H. Painter (S'62-M'63-SM'72) received the B.S. and M.S. degrees from the University of Illinois, Urbana, did graduate work in mathematics at the College of William and Mary, Williamsburg, VA and received the Ph.D. degree at Southern Methodist University, Dallas, TX.

He has been an Air Force Officer/Navigator and a NASA Communication Engineer, working on the Apollo Moon Project. He is a Professor of Electrical Engineering at Texas A \& M University, College Station, where he has been active for the last five years in the planining and managing of computing activities. He is currently directing the development of an engineering computing facility which will support the university's pilot project in computer-based education. $\mathrm{He}$ is an author and inventor, holding patents on digital data communication devices. He is currently active in the U.S. and abroad as a lecturer and consultant in satellite radio-navigation systems.

Dr. Painter is a member of five technical and honorary societies, a Registered Professional Engineer, and a private pilot.

\title{
Frequency-Selective Fading Effects in Digital Mobile Radio with Diversity Combining
}

\author{
BERNARD GLANCE, MEMBER, IEEE, AND LARRY J. GREENSTEIN, SENIOR MEMBER, IEEE
}

\begin{abstract}
We analyze the effects of frequency-selective fading in a cellular mobile radio system that uses 1) phase-shift keying (PSK) with cosine rolloff pulses, and 2) space diversity with maximal-radio combining. The distorting phenomena with which we deal are multipath fading (which produces the frequency selectivity), shadow fading; and cochannel interference. The relevant quality measure is defined to be the bit error rate averaged over the multipath fading, denoted by 〈BER $\rangle$. The relevant system performance characteristic is defined to be the probability distribution for $\langle$ BER $\rangle$, taken over the ensemble of shadow fadings and locations of the desired and interfering mobiles.

To obtain numerical results, we use a combination of analysis and Monte Carlo simulation, invoke widely accepted models for the multipath and shadow fadings, and assume a cellular system with seven channel sets and centrally located base stations. The outcome is a set of performance curves that reveal the influences of various system and channel parameters. These include: the number of modulation levels (two or four), the diversity order, the shape of the multipath delay spectrum, and the standard deviation (or delay spread, $\tau_{0}$ ) of the multipath delay spectrum. Practical factors accounted for in these assessments include fading- and interference-related timing recovery errors and combiner imperfections.

Our results highlight the importance of the ratio $\tau_{0} / T$, where $T$ is the digital symbol period. They show that the delay spectrum shape is of no importance for $\tau_{0} / T \leq 0.2$, but can have a profound influence for $\tau_{0} / T \geq 0.3$. We also find that using 4-PSK leads to better detection performance, in certain cases, than using 2-PSK.
\end{abstract}

Paper approved by the Editor for Data Communication Systems of the IEEE Communications Society for publication without oral presentation. Manuscript received September 29, 1982; revised March 7, 1983.

The authors are with the Crawford Hill Laboratory, Bell Laboratories, Holmdel, NJ 07733.

\section{INTRODUCTION}

$I^{\mathrm{N}}$ $N$ the digital mobile radio environment, transmission between mobiles and base stations takes place over multipath channels. The associated delay spreads cause frequencyselective fading, which limits system performance by causing intersymbol interference in the detection process.

The effects of frequency-selective fading in digital radio communication were extensively analyzed by Bello and Nelin [1] for binary differentially encoded signals using square pulse signaling. They assumed an impulse response that is slowly time-varying, where the value for any delay is a complex Gaussian stationary process and is independent of the values at all other delays. Such a chaninel can be characterized, in part, by the mean-square magnitude of the impulse response; ${ }^{1}$ we call this function the delay spectrum, and scale it in amplitude so that its area is unity.

Lacking specific data on the shape of the delay spectrum, Bello and Nelin assumed a Gaussian shape. Bailey and Lindenlaub [2] extended the analysis to Nyquist pulse signaling and assumed a square-shaped delay spectrum. Both studies were made assuming a matched filter receiver with linear diversity

1 A complete characterization requires, in addition, a description for the temporal variations of the impulse response. In the present study, wherein the signaling rates are large compared to the fading rates, this aspect of the channel will not be treated explicitly. Instead, we will analyze the channel response as though random but static, and perform appropriate averages over the randomly changing responses. 